Document downloaded from:

http://hdl.handle.net/10251/151035

This paper must be cited as:

Parcero Iglesias, E.; Flores, L.; Sánchez, MG.; Vidal-Gimeno, V.; Verdú Martín, GJ. (2017). Impact of view reduction in CT on radiation dose for patients. Radiation Physics and Chemistry. 137:173-175. https://doi.org/10.1016/j.radphyschem.2016.01.038

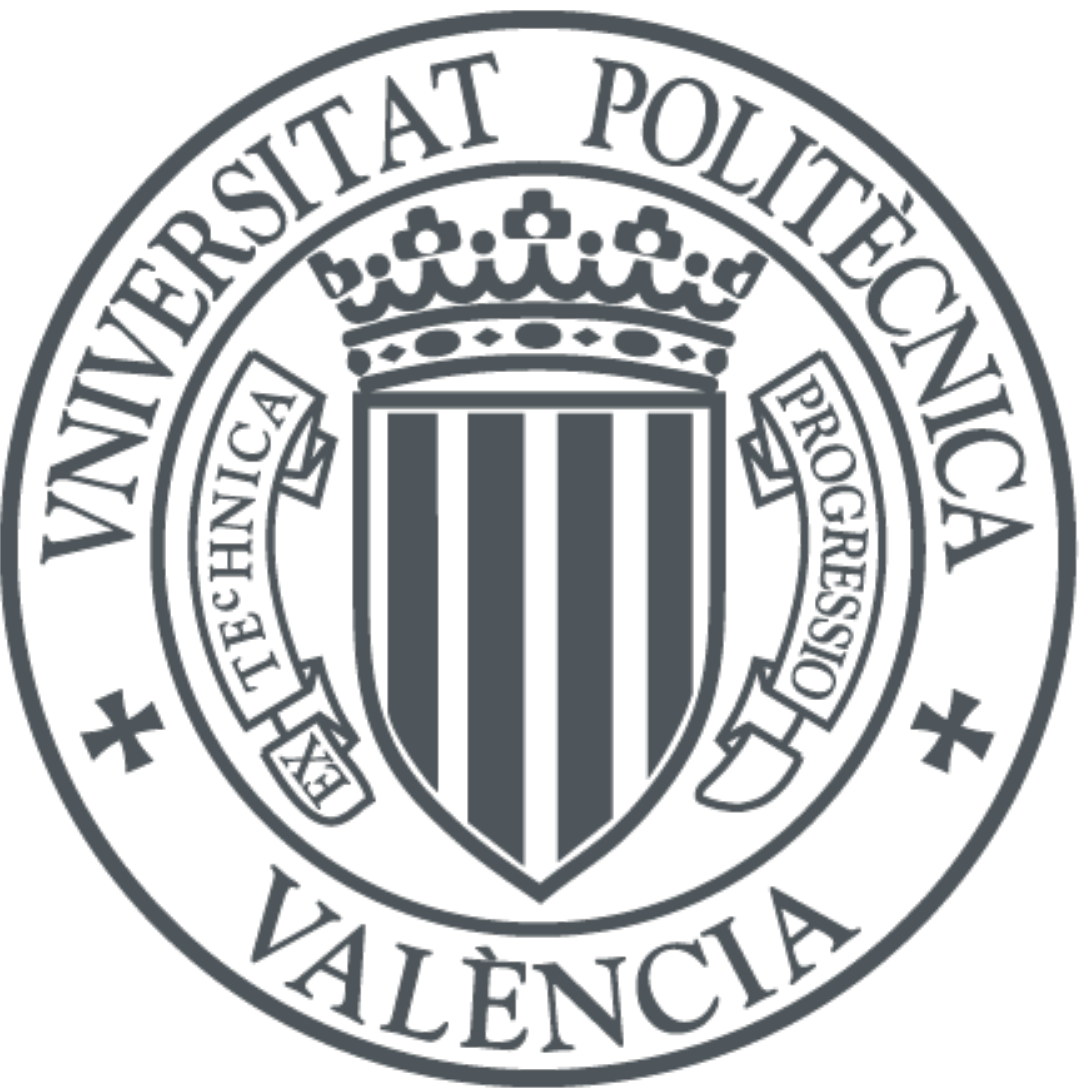

The final publication is available at

https://doi.org/10.1016/j.radphyschem.2016.01.038

Copyright Elsevier

Additional Information 


\title{
Impact of View Reduction in CT on Radiation Dose for Patients
}

\author{
V. Vidal ${ }^{a, *}$, E. Parcero ${ }^{\mathrm{b}, *}$, L. Flores ${ }^{\mathrm{c}}$, M.G. Sánchez ${ }^{\mathrm{d}}$, G. Verdú ${ }^{\mathrm{b}}$ \\ ${ }^{a}$ DSIC, Universitat Politècnica de València, Cno Vera $s / n$ 46022, Valencia, Spain \\ ${ }^{b}$ ISIRYM, Universitat Politècnica de València, Cno Vera s/n 46022, Valencia, Spain \\ ${ }^{c}$ Universidad Peruana Cayetano Heredia, Av Honorio Delgado 340, 15120, Lima, Peru \\ ${ }^{d}$ Instituto Tecnológico, Cd Guzmán, Jalisco, México
}

\begin{abstract}
Iterative methods have become a hot topic of research in computed tomography (CT) imaging because of their capacity to resolve the reconstruction problem from a limited number of projections. This allows the reduction of radiation exposure on patients during the data acquisition. The reconstruction time and the high radiation dose imposed on patients are the two major drawbacks in CT. To solve them effectively we adapted the method for sparse linear equations and sparse least squares (LSQR) with soft threshold filtering (STF) and the fast iterative shrinkage-thresholding algorithm (FISTA) to computed tomography reconstruction. The feasibility of the proposed methods are demonstrated numerically.
\end{abstract}

Keywords: Nuclear medicine CT, Noise reduction, Few-view

\section{Introduction}

In Computer Tomography (CT) imaging, a set of projections taken with a scanner is used to reconstruct the internal structure of an object. Analytical methods based on the inverse Fourier transform require the complete data collection which is not always possible to obtain(Deans, 2007; Herman, 2009). Also, they do not provide the optimal reconstruction in images with noise (Wang et al., 2008).

Iterative algorithms have drawn much attention in medical imaging because of their capacity to solve the reconstruction problem from a limited number of projections, also known as few-view reconstruction (Beister et al., 2012; Zhao et al., 2013; Flores et al., 2013, 2014; Donoho, 2006; Yu and Wang, 2010; Yu and Zeng, 2014). This allows the possibility of reducing radiation exposure on patients during the data acquisition. Many algorithms have been developed in the field of few-view CT image reconstruction. In $\mathrm{CT}$, it is common to find an incomplete set projections. In these cases, iterative methods allow to reconstruct images with higher contrast and precision in noisy conditions from a

\footnotetext{
*Corresponding authors

Email addresses: vvidal@dsic.upv.es (V. Vidal), esparig@upvnet.upv.es (E. Parcero)
} 
small number of projections and provide images with better quality. However, the major drawback of these methods is given by their high computational cost.

A diagnosis based on $\mathrm{CT}$ is fundamental for the detection of abnormal tissues by different attenuation of X-rays, which, frequently, are not clearly distinguished by radiologists. However, excessive X-ray radiation exposure is undesirable. In this work, we study CT image reconstruction using few number of projections to be able to reduce the absorbed radiation dose by the patient.

Inspired by these methods and with the aim of reducing computational cost while preserving the quality of the reconstructed image, we propose a method based on LSQR(Paige and Saunders, 1982) with STF (Yu and Wang, 2010) (soft threshold filtering) and the FISTA (fast iterative shrinkage-thresholding) algorithm (Beck and Teboulle, 2009).

The paper is organized as follows: in section Methods, relevant mathematical aspects of the methods used are presented, as well as how the CT data was acquired, and the metrical measures employed in the study. After that, we describe the methodology used to carry out a quantitative analysis and present results of the implementation of these algorithms. Finally, we summarize our conclusions.

\section{Methods}

Algebraically, the reconstruction problem from a limited number of projections is reduced to solving a linear system of the form $A x=b$, where $b=\left[b_{1}, b_{2}, \ldots, b_{M}\right]^{T} \in R^{M}$ is a column vector containing the $M$ obtained projections (number of rays $\times$ number of views), $x=\left[x_{1}, x_{2}, \ldots, x_{N}\right]^{T} \in R^{N}$ is the image to be reconstructed where $N$ is the number of pixels, and $A=\left(a_{i j}\right) \in R^{M x N}$ is the system matrix which represents the forward projection. In this approach, to reconstruct the internal structure of an object is equivalent to solving equation above in terms of measured projections.

LSQR solves the system equation by minimizing $\|b-A x\|_{2}$ within a sequence of Krylov subspaces (Golub and Kahan, 1965). This technique generates a sequence of approximations $\left\{x_{k}\right\}$ such that the residual norm $\left\|r_{k}\right\|_{2}$, where $r_{k}=b-A x_{k}$, decreases monotonically.

With the aim of eliminating undesired artifacts and preserving the edge structure of the object, we adopted a soft threshold filtering (STF) approach for reconstruction from a limited number of projections (Yu and Wang, 2010). While the STF technique helps to preserve the edge data structure of the object, the FISTA algorithm introduces new directions of the solution that are used by LSQR to accelerate the convergence and to improve the image quality. This technique is similar to (Beck and Teboulle, 2009).

Our method combines LSQR with STF and FISTA. As we mentioned above, this combination speedups the convergence process and eliminates undesired artifacts.

To evaluate the performance of the algorithms the following quality metrics are employed: Mean Absolute Error (MAE), Mean Square Error (MSE), Peak Signal-to-Noise Ratio (PSNR), and Structural SIMilarity (SSIM).

PSNR is used to measure noise reduction and MAE is used for the preservation of the signal. To define the PSNR, we need to calculate the MSE, which gives a more complete idea of the overall error.

Lastly, the SSIM score of the entire image is computed by simply averaging some measured properties of the image. SSIM scores are much more consistent than the MSE 
Table 1: Evaluation of the reconstruction with LSQR-STF-FISTA employing 45 views after 2000 iterations

\begin{tabular}{lcccc}
\hline & Noise-free & Gaussian $(0.01)$ & Gaussian $(0.05)$ & Gaussian $(0.1)$ \\
\hline \hline MAE & 0.0003 & 0.0021 & 0.0100 & 0.0188 \\
\hline MSE & 0.0000 & 0.0000 & 0.0001 & 0.0006 \\
\hline PSNR & 69.026 & 51.209 & 37.839 & 32.200 \\
\hline SSIM & 0.9997 & 0.9601 & 0.6895 & 0.6035 \\
\hline
\end{tabular}

scores relative to visual perception, and its maximum value is 1 , achieved only if the two compared images are the same.

To determine the optimal parameters of the proposed method we performed simulations with the FORBILD head phantom. The FORBILD head phantom is a sophisticated mathematical phantom widely seen as an adequate gold standard (Yu et al., 2012). We generated projections of a typical slice of the phantom of size $256 \times 256$ using a CT with 1025 virtual detectors. The pixel size of the reconstructed image would be $0.1 \times 0.1 \mathrm{~cm}^{2}$. The radiation source takes a projection for each degree of rotation. Subsets of 36, 45, 90, and 180 projections are composed from the 360 obtained projections. Some Gaussian noise (variance set to $0.01,0.05$, and 0.1 ) is added to the projections to test the reconstruction capacity of the described methods.

\section{Results and Discussion}

The evaluation performed seeks to compare the different methods of reconstruction (LSQR, LSQR-STF, and LSQR-STF-FISTA) using different collections of projections that vary in number of projections and noise acquired. The comparison is made using the previously described quality metrics.

Figure 1 shows the reconstruction results in terms of PSNR scores, comparing the different methods and using different number of views (180, 90, 45, and 36). LSQR needs a higher number of iterations to obtain good results. LSQR-STF and LSQR-STF-FISTA improve convergence, achieving good PSNR scores even with fewer views. Among the considered methods, FISTA is the only one that get good enough results with 45 and 36 views. The fewer the number of views, the worse the results are, and the slower the convergence.

Table 1 shows the results of reconstruction with the different collections of 45 views (noise-free and Gaussian noise). The SSIM obtained with the noise-free projections indicates an almost perfect reconstruction, and a very good reconstruction with little Gaussian noise (variance $=0.01$ )

Despite the numerical results seems to be not very promising for high-variance noise, the reconstructed images are sufficiently clear to distinguish the different structures of the phantom. In presence of low-variance noise the reconstruction is free of artifacts, achieving a high-quality image.

\section{Conclusions}

It has been tested the LSQR method in combination with the STF technique and the FISTA algorithm in the reconstruction of images from 36, 45, 90, and 180 views using 

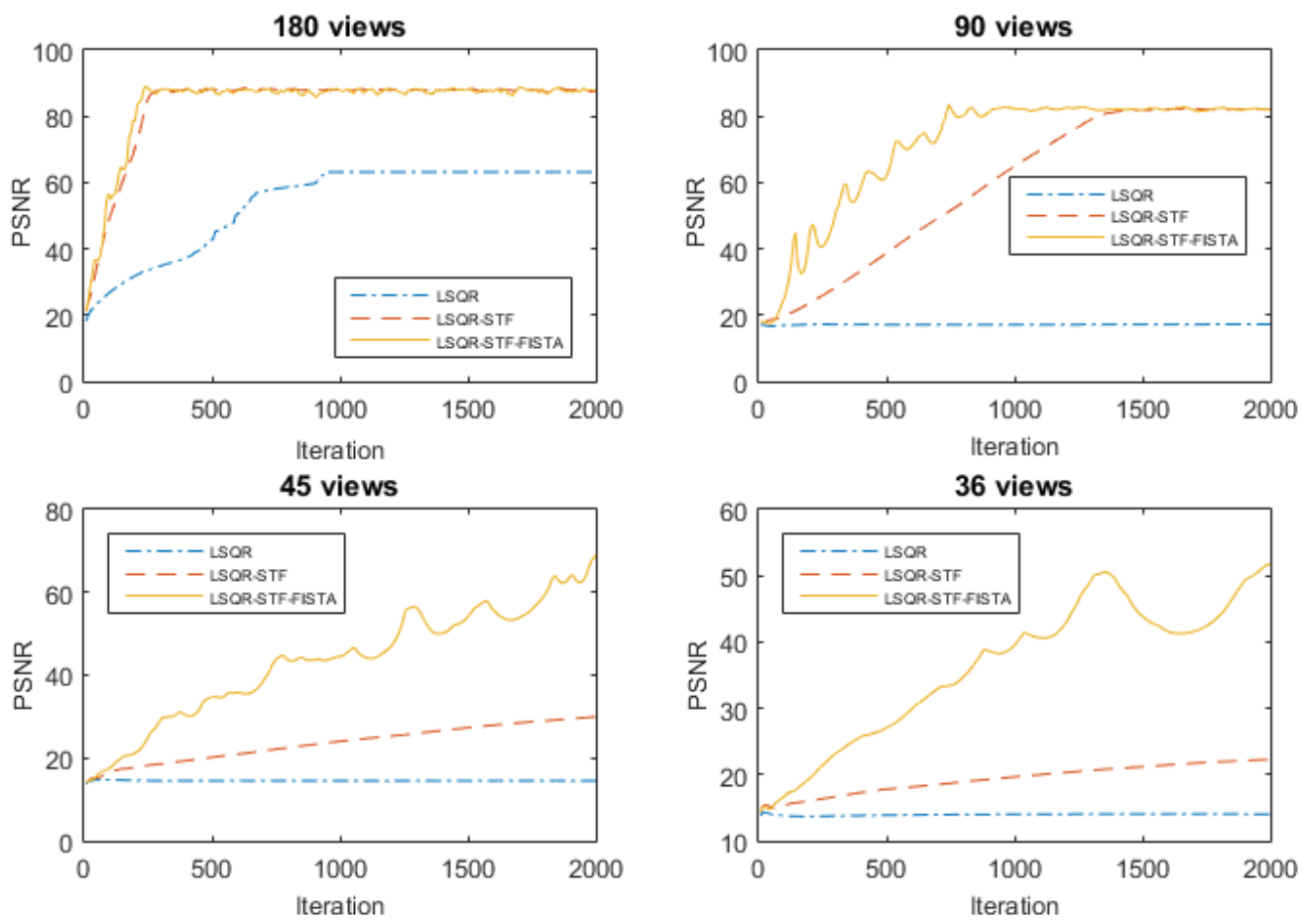

Figure 1: Comparison between methods, for each collection of noise-free projections $(180,90,45$, and 36 views)

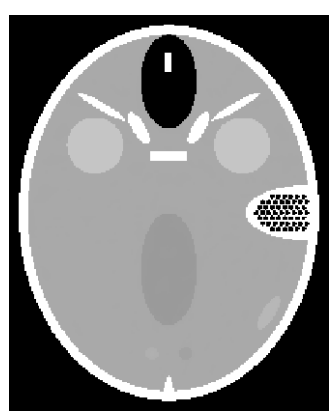

Noise-free

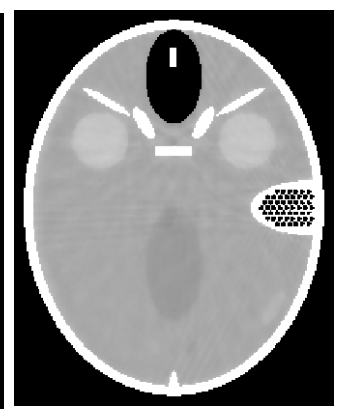

Gaussian (0.01)

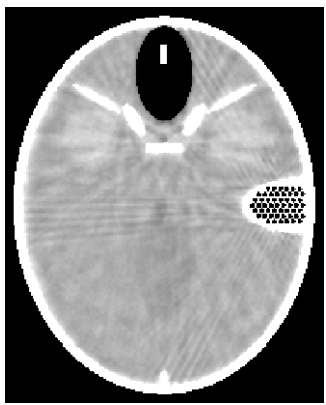

Gaussian (0.05)

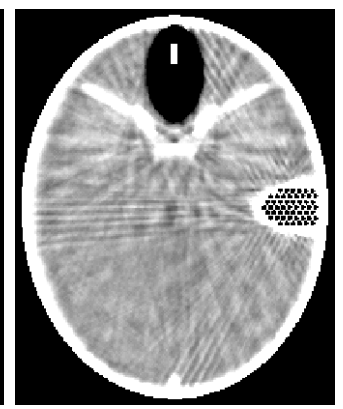

Gaussian (0.1)

Figure 2: Images reconstructed by LSQR-STF-FISTA using 45 views after 2000 iterations from noisefree projections (first row), and projections with Gaussian noise (variance equal 0.01, 0.05, and 0.1 in following rows). 
noisy-free projections showing better results than LSQR-STF and LSQR alone: values of PSNR above 80 for 180 and 90 projections, and above 50 for 45 and 36 projections.

Comparing the obtained results, it is observed that the reconstructions from 180 views after 400 iterations have a good quality, with a PSNR near 85 . The reduction of views to 45 and even to 36 is possible especially if the projections are noise-free, leading to a reconstruction without or few artifacts.

In presence of noise the reconstruction is possible using the LSQR-STF-FISTA method. Low noise signals can be almost suppressed, obtaining a SSIM of 0.96. Higher signals do not prevent the reconstruction, and the phantom structures are visible, although possibly blurred (SSIM below 0.70).

The possibility of reconstructing images from a limited number of projections allows a reduction of time in the data acquisition process, as well as the radiation exposure in the patients.

\section{References}

Andersen, A., Kak, A. C., 1984. Simultaneous algebraic reconstruction technique (sart): a superior implementation of the art algorithm. Ultrasonic imaging 6 (1), 81-94.

Beck, A., Teboulle, M., 2009. A fast iterative shrinkage-thresholding algorithm for linear inverse problems. SIAM journal on imaging sciences 2 (1), 183-202.

Beister, M., Kolditz, D., Kalender, W. A., 2012. Iterative reconstruction methods in x-ray ct. Physica medica 28 (2), 94-108.

Candès, E. J., 2006. Compressive sampling. In: Proceedings of the international congress of mathematicians. Vol. 3. pp. 1433-1452.

Daubechies, I., Defrise, M., De Mol, C., 2003. An iterative thresholding algorithm for linear inverse problems with a sparsity constraint. arXiv preprint math/0307152.

Daubechies, I., Fornasier, M., Loris, I., 2008. Accelerated projected gradient method for linear inverse problems with sparsity constraints. Journal of Fourier Analysis and Applications 14 (5-6), 764-792.

Deans, S. R., 2007. The Radon transform and some of its applications. Dover Publications Inc.

Donoho, D. L., 2006. Compressed sensing. Information Theory, IEEE Transactions on 52 (4), 1289-1306.

Flores, L. A., Vidal, V., Mayo, P., Rodenas, F., Verdú, G., 2013. Ct image reconstruction based on gpus. Procedia Computer Science 18, 1412-1420.

Flores, L. A., Vidal, V., Mayo, P., Rodenas, F., Verdú, G., 2014. Parallel ct image reconstruction based on gpus. Radiation Physics and Chemistry 95, 247-250.

Golub, G., Kahan, W., 1965. Calculating the singular values and pseudo-inverse of a matrix. Journal of the Society for Industrial \& Applied Mathematics, Series B: Numerical Analysis 2 (2), $205-224$.

Herman, G., 2009. Fundamentals of Computerized Tomography: Image Reconstruction from Projections. Advances in Computer Vision and Pattern Recognition. Springer. URL https://books.google.es/books?id=BhtGTkEjkOQC

Paige, C. C., Saunders, M. A., 1982. Lsqr: An algorithm for sparse linear equations and sparse least squares. ACM Transactions on Mathematical Software (TOMS) 8 (1), 43-71.

Wang, G., Yu, H., De Man, B., 2008. An outlook on x-ray ct research and development. Medical physics 35 (3), 1051-1064.

Wang, Z., Bovik, A. C., Sheikh, H. R., Simoncelli, E. P., 2004. Image quality assessment: from error visibility to structural similarity. Image Processing, IEEE Transactions on 13 (4), 600-612.

Yu, H., Wang, G., 2010. A soft-threshold filtering approach for reconstruction from a limited number of projections. Physics in medicine and biology 55 (13), 3905.

Yu, W., Zeng, L., 2014. A novel weighted total difference based image reconstruction algorithm for few-view computed tomography.

Yu, Z., Noo, F., Dennerlein, F., Wunderlich, A., Lauritsch, G., Hornegger, J., 2012. Simulation tools for two-dimensional experiments in x-ray computed tomography using the forbild head phantom. Physics in medicine and biology 57 (13), N237.

Zhao, X., Hu, J.-J., Yang, T., 2013. Gpu based iterative cone-beam ct reconstruction using empty space skipping. Journal of X-ray science and technology 21 (1), 53-69. 